\title{
Peptide-Mediated Constructs of Quantum Dot Nanocomposites for Enzymatic Control of Nonradiative Energy Transfer
}

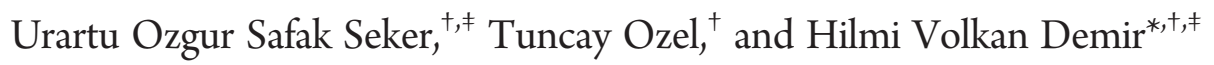 \\ ${ }^{\dagger}$ Department of Electrical and Electronics Engineering, Department of Physics and UNAM-Institute of Materials Science and \\ Nanotechnology, Bilkent University, 06800 Turkey \\ ${ }^{\ddagger}$ Luminous! Centre of Excellence for Semiconductor Lighting and Displays, School of Electrical and Electronic Engineering, \\ Microelectronics Division, School of Physical and Mathematical Sciences, Physics and Applied Physics Division, \\ Nanyang Technological University, 639798 Singapore
}

Supporting Information

\begin{abstract}
A bottom-up approach for constructing colloidal semiconductor quantum dot (QDot) nanocomposites that facilitate nonradiative Förster-type resonance energy transfer (FRET) using polyelectrolyte peptides was proposed and realized. The electrostatic interaction of these polypeptides with altering chain lengths was probed for thermodynamic, structural, and morphological aspects. The resulting nanocomposite film was successfully cut with the protease by digesting the biomimetic peptide layer upon which the QDot assembly was constructed. The ability to control photoluminescence decay lifetime was demonstrated by proteolytic enzyme activity, opening up new possibilities for biosensor applications.
\end{abstract}

KEYWORDS: Nanocomposites, quantum dots, polypeptides, proteolytic, nonradiative energy transfer, nanobiotechnology
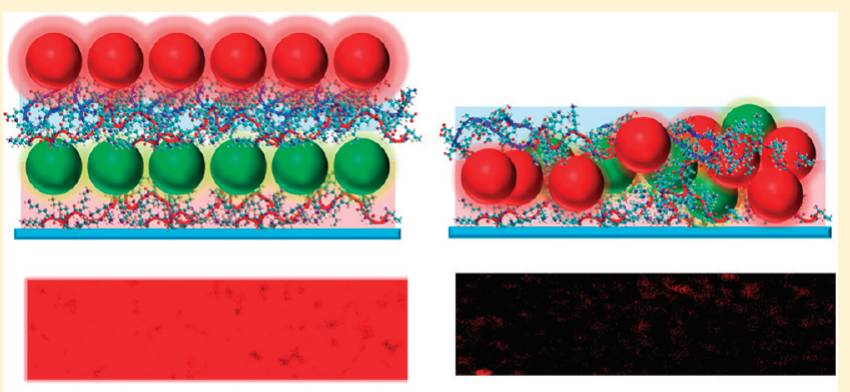

Intact Peptide Film Assembly of QDots

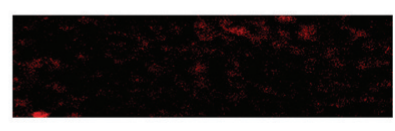

After Protease Attack
$\mathrm{B}$ iomolecules, especially proteins, peptides, and DNA, which function as the molecular building blocks of biological systems, are of fundamental importance for creating future biogeneration of electronic and photonic devices and sensors in hybrid architectures. ${ }^{1-4}$ Their unique molecular capabilities in self-assembly make these molecules desired candidates for hybrid applications at the nano-bio interface (compared to available synthetic approaches). In nanotechnology applications, great attention has been paid to biohybrid nanomaterial systems to discover new assembly techniques. ${ }^{5-9}$ In this context, the interaction of nanoparticles with proteins, peptides, and DNA has been studied for innovative and robust biohybrid designs. ${ }^{10-12}$

Colloidal semiconductor quantum dots (QDots), also known as nanocrystals, have been utilized in several nano- and biotechnological applications. ${ }^{13,14}$ Their attractive electronic and optical properties including size-tunable optical emission, high quantum yield, and photostability enabled these nanoparticles to be exploited in various photonic device platforms. ${ }^{15}$ In recent studies, for example, capabilities of QDots have been demonstrated for white light generation and tunability in color-conversion light-emitting diodes. ${ }^{16,17}$ With their unique and desirable properties QDots have been one of the most widely studied classes of nanomaterial systems for protein and peptide based systems. ${ }^{18}$ Quantum dot labeled proteins and peptides promise to be useful as molecular probes in many applications including cancer targeting, ${ }^{19,20}$ determination of toxins in food and environmental samples, ${ }^{21}$ and detection and quantification of pathogenic microorganisms in food samples. ${ }^{22}$ Beyond sensitive biolabeling applications, in the past decade, studies also showed that semiconductor QDots (e.g., CdTe and CdSe) can be utilized in Förster-type resonance energy transfer (FRET) processes. Such nonradiative energy transfer directs excitation energy from donor QDots to acceptor QDots in close proximity. $^{23-25}$

QDots have been widely used in FRET processes in biomolecular systems and many successful applications were demonstrated. In the work reported by Matussi et al., maltose binding proteins labeled with Cy3 dye (emitting at $570 \mathrm{~nm}$ ) were conjugated to QDots emitting at $510 \mathrm{~nm}$, and the resulting energy transfer between the dye molecules and quantum dots was probed. Upon binding the proteins changed conformation to allow FRET. ${ }^{26}$ Similar studies were also conducted for the targeted conjugation of QDots with proteins without sacrificing the protein functionality. ${ }^{27}$ In another study QDot-protein bioconjugates were utilized in building protein arrays for specific recognition of targeted antibodies and elements, where FRET signal was used for robust detection of protein-antigen molecule interaction. ${ }^{28,29}$ Quenching is another phenomenon used as a signal from QDot-protein bioconjugates. This was exploited to detect a drug molecule

Received: December 9, 2010

Revised: $\quad$ March 7, 2011

Published: March 23, 2011 

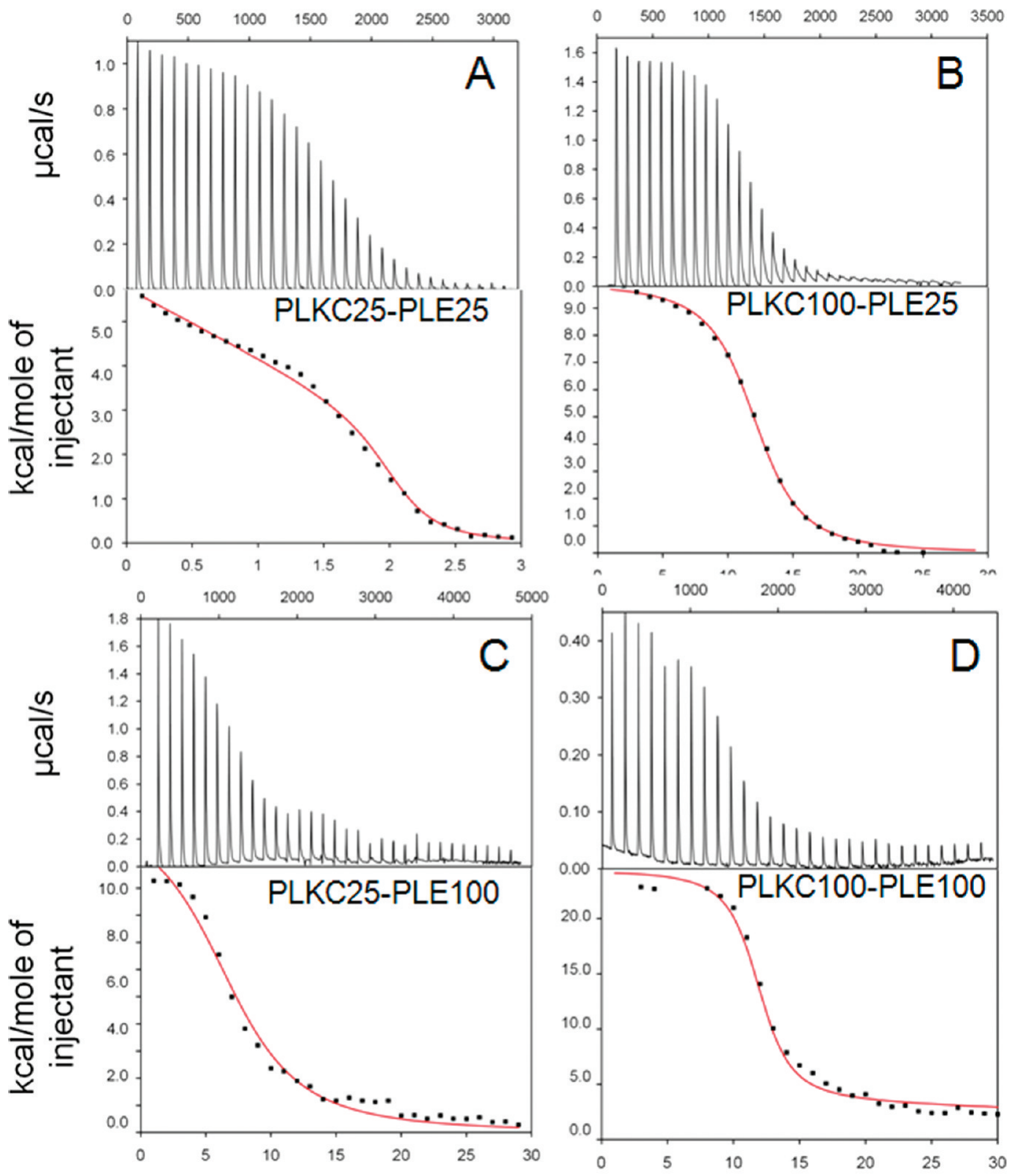

Molar Ratio $\left(\mathrm{X}_{\mathrm{t}}\right)$

Figure 1. Isothermal titration calorimetry (ITC) curves for the peptide pairs: PLKC25-PLE25 (A), PLKC100-PLE25 (B), PLKC25-PLE100 (C), and PLKC100-PLE100 (D). In the top graphs, the black solid line shows the actual experimental data of titration peaks for each sample. In the bottom graphs, the red solid line represents the model fitted to the experimental data to extract $\Delta H, \Delta S$, and $K_{\text {eq }}$, while the integrated peak areas are given in the black dotted line.

named paraoxon, in the context of which a QDot-protein based detector was proposed. ${ }^{30}$ FRET applications of QDot bioconjugates were also integrated in microfludic systems; this concept was introduced in a proof-of-concept study toward detection of DNA, where QDot-functionalized oligomers were successfully employed. ${ }^{31}$

To design and implement solid state devices using QDots, however, thin film concept is essential. Thin film approach allows for forming nanostructures with film thicknesses ranging from nanometers to micrometers. There are a number of different methods for thin film construction including spin coating ${ }^{32,33}$ and layer-by-layer (LbL) assembly. ${ }^{34}$ In LbL assembly molecules can be piled up in a sequential arrangement with desired spatial distribution. Because the number of separation layers can control the distance between active layers, $\mathrm{LbL}$ film is a perfect architecture to study FRET applications. ${ }^{35}$

Layer-by-layer deposition of inorganic materials in biological systems is common. Lustrin is a protein with polycationic domains, managing formation of layer-by-layer assembly of aragonite in mollusk shell, ${ }^{36,37}$ and similarly silicatein protein is responsible for the formation and assembly of complex silica structures in a diatom, Cylindrotheca fusiformis. Molecular characterization of silicatein protein validated the existence of polyelectrolyte peptide domains that are effective in silica formation. ${ }^{38}$ Similarly, prismatic asprich protein family is located in the shell matrix of the mollusk shell and, through the electrostatic calcium binding domain, this mediates the layer- 
Table 1. Thermodynamic Constants Gathered from Isothermal Titration Calorimetry (ITC) Experiments for Each of the Peptide Pairs ${ }^{a}$

\begin{tabular}{cccc} 
peptide couple & \multicolumn{3}{c}{$\Delta S\left(\mathrm{cal} \mathrm{mol}^{-1}\right.$} \\
\cline { 2 - 2 } & $24.0 \pm 0.5$ & 113.0 & $(6.9 \pm 1.5) \times 10^{6}$ \\
PLKC 100-PLE 25 & $10.0 \pm 0.8$ & 58.6 & $(3.3 \pm 1.4) \times 10^{5}$ \\
PLKC 25-PLE 100 & $12.0 \pm 0.6$ & 62.9 & $(7.9 \pm 1.4) \times 10^{4}$ \\
PLKC 25-PLE25 & $5.7 \pm 0.2$ & 46.5 & $(2.2 \pm 0.3) \times 10^{5}$
\end{tabular}

${ }^{a}$ The ITC data were analyzed and fit to the model using Origin 7 supplied with ITC200.

by-layer deposition of calcium carbonate. ${ }^{39}$ The asprich protein family contains high amounts of polycationic residues, and these polyelectrolyte domains are assigned for the $\mathrm{LbL}$ assembly of the inorganic calcium. ${ }^{40}$ The strength and robustness of the layer-by-layer assembled biomineral matrices provides us with a favorable model to construct LbL-assembled QDots for FRET applications.

In this paper we proposed and demonstrated QDot nanocomposites constructed using polyelectrolyte peptides, poly-Llysine (PLKC), and poly-L-glutamic acid (PLE), with varying chain lengths to stack QDots for FRET processes. First, we characterized the binding and structural rearrangements of these peptides upon their interactions with each other. After the assembly of peptides, adsorption of donor CdTe QDots emitting at $560 \mathrm{~nm}$ on the peptide layer was achieved and varying numbers of peptide interlayers were subsequently formed, on top of which acceptor CdTe QDots emitting at $640 \mathrm{~nm}$ were placed. Using a quartz crystal microbalance, the change in film thickness was tracked upon deposition of sequential layers. The peptide films were also characterized using atomic force microscopy, for comparison of all possible combinations of PLKC and PLE (with varying chain lengths of 100 and 25). The peptide film combinations were tested for the energy transfer using both steady-state and time-resolved photoluminescence spectroscopy. For its superior film quality, PLKC100-PLE25 was studied for assays with protease. Its nanocomposite films with QDots were successfully cut by digesting biomimetic peptide layers with the protease. The proteolytic enzyme control of the acceptor lifetimes in these QDot-peptide constructs was demonstrated. Such peptide-mediated QDot solids are promising for novel biologically controlled devices and sensors.

To form peptidic thin films for the assembly of QDots, poly-Llysine and poly-L-glutamic acid were custom synthesized by Alamanda Polymers (AL, USA), using solid-state peptide synthesis with a purity of $95 \%$. The peptides were synthesized with $25 \mathrm{mer}$ and $100 \mathrm{mer}$ chain lengths; no further purification was made (see Supporting Information). To probe the effect of different peptide chain lengths on the assembly of the peptide film, the thermodynamics of interaction between the peptides was investigated. The binding of positively charged PLKC molecules and negatively charged PLE molecules was monitored by using an isothermal titration calorimetry (ITC), ITC200, from Microcal (GE Healthcare, Austria), under continuous stirring at $1000 \mathrm{rpm}$ with thermostating at $25^{\circ} \mathrm{C}$. The binding isotherms were fit to a two-site binding model to extract the thermodynamic parameters, where the initial points were discarded to avoid possible errors caused by the initial diffusion of injectant in each isotherm. The data were analyzed and fit to the model using the software package Origin 7 supplied with ITC200 (see Supporting Information). PLKC molecules, which have either 25 or 100 amino acid (aa) chain lengths, were titrated against the negatively charged PLE molecules, again with the chain lengths of 25 or 100 aa, in all possible combinations. The areas under the thermodynamic titration peaks were integrated and the thermodynamic parameters were calculated by fitting these peak areas to the interaction model in Origin 7. Figure 1 shows experimental thermodynamic titration curves (in black), along with their data fittings (in red), and Table 1 lists the thermodynamic constants (enthalpy changes, entropy changes, and binding equilibrium constants) obtained from these ITC experiments for each of the peptide pairs.

The peptide chain length controls the binding energy and affinity through the functional side chains. However, given a peptide, there is no simple linear relationship. ${ }^{41}$ In this case here, we have concatamers of the same amino acid, for which we expect an increased binding affinity with the increasing peptide chain length. In our analysis, the interaction of PLKC100PLE100 was found to have the highest binding energy, while PLKC25-PLE25 has the lowest binding affinity among all peptide combinations. The other peptide combinations, PLKC100PLE25 and PLKC25-PLE100, expectedly exhibit their binding energies between the highest and lowest levels. The interaction of each peptide has a positive enthalpy change, which indicates that the interaction of the peptides is an endothermic process.

A large entropy change with a positive sign is also notable for the interactions. The positive entropy may arise due to the loss of the structured water shell formed around the peptides upon peptide-peptide interaction. Peptides are surrounded by a water shell in buffer, and the charge of peptides and salt ions rules the formation of a water shell around the peptide molecules. However, when two oppositely charged peptides are interacting, the water in the contact surface of the peptides is repelled. This causes the rearrangement of contact sides of the peptides. This is generally attributed to the electrostatic interaction and burial of the polar groups during protein-protein interaction. $^{42-44}$ Compared to the other peptide pairs, the PLKC100-PLE100 pair features the highest equilibrium binding constant, which is $\sim 6.9 \times 10^{6} \mathrm{M}^{-1}$, and, surprisingly, the change in affinity constant of the peptide pairs does not follow a trend. PLKC100-PLE25 and PLKC25-PLE100 do not exhibit a similar level of binding capability, with the binding constant of PLKC100-PLE25 $\left(\sim 3.3 \times 10^{5} \mathrm{M}^{-1}\right)$ being more than 4-fold better than that of PLKC25-PLE100 $\left(\sim 7.9 \times 10^{4} \mathrm{M}^{-1}\right)$. It was also unexpected that the shortest chain combination leads to an intermediate-level interaction constant of $\sim 2.2 \times 10^{5} \mathrm{M}^{-1}$, which is larger than PLKC25-PLE100 and smaller than PLKC100-PLE25.

Next, the structural investigation of the peptide pairs is important because the structure of these peptides directs their binding affinity during film formation on a given surface. ${ }^{45}$ Here the secondary structures of the peptides were investigated using circular dichroism (CD) spectroscopy presented in Figure 2. $\mathrm{CD}$ measurements were taken using peptides with $25 \mu \mathrm{M}$ concentrations dissolved in phosphate buffer $(\mathrm{pH} 7.4)$ in a quartz cuvette with $1 \mathrm{~mm}$ path length (see Supporting Information). The data were deconvoluted using a package program called CDPro. ${ }^{46}$ The program uses a large protein secondary structure database as a reference set. By employing a least-squares curve fitting algorithm, the program calculates the amount of the secondary structure in a given sample on the basis of the chosen reference set. It is thus 


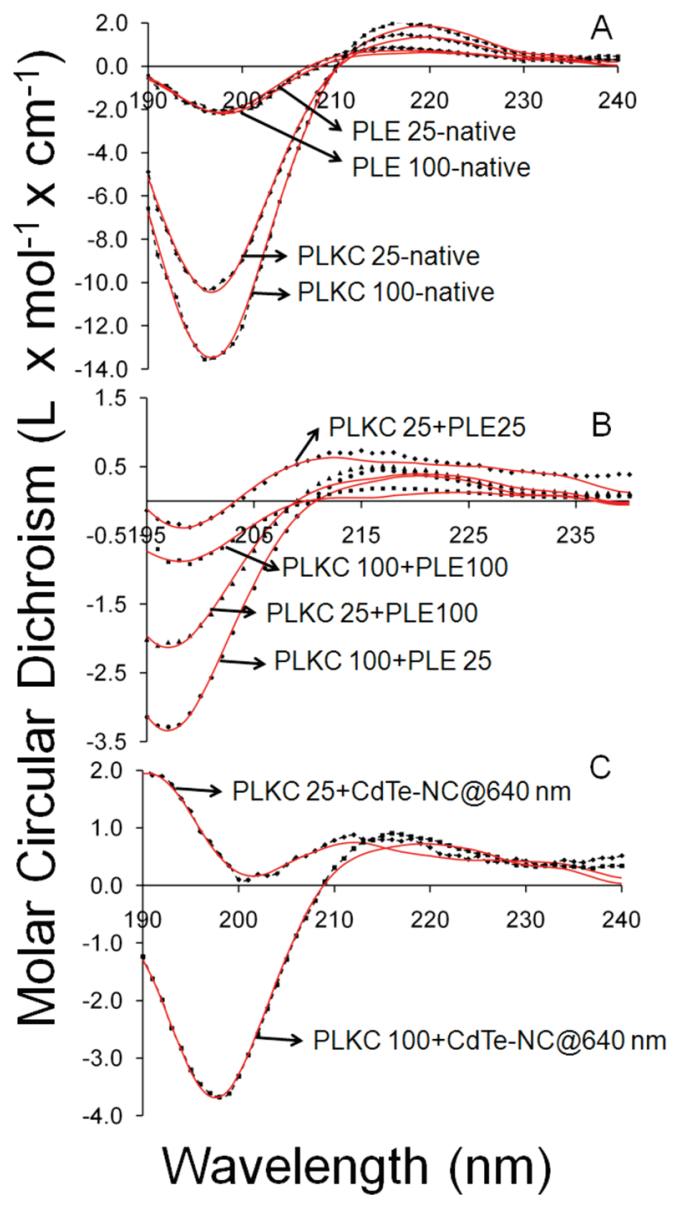

Figure 2. Circular dichroism (CD) spectra for individual peptides (A), peptide pairs interacting each other (B), and peptides interacting with CdTe QDots (C). CDPro program-based secondary structure database was used to fit the experimental data (given in black dotted line) using the least-squares curve fitting (shown in red solid line). Each sample is labeled on the data line.

Table 2. Secondary Structure Elements of Individual Peptides, Peptide Pairs, and Peptides Interacting with CdTe QDots. $^{a}$

\begin{tabular}{lcccc} 
& \multicolumn{4}{c}{ secondary structure elements } \\
\cline { 2 - 5 } \multicolumn{1}{c}{ peptides } & helical & strand & turn & unordered \\
PLKC100 & 0.06 & 0.23 & 0.20 & 0.51 \\
PLKC25 & 0.17 & 0.15 & 0.24 & 0.45 \\
PLE100 & 0.04 & 0.42 & 0.20 & 0.35 \\
PLE25 & 0.04 & 0.41 & 0.20 & 0.35 \\
PLKC100-PLE100 & 0.04 & 0.41 & 0.21 & 0.34 \\
PLKC100-PLE25 & 0.04 & 0.42 & 0.22 & 0.33 \\
PLKC25-PLE100 & 0.04 & 0.40 & 0.21 & 0.35 \\
PLKC25-PLE25 & 0.03 & 0.44 & 0.20 & 0.33 \\
PLKC100-QDot @ 640 nm & 0.04 & 0.38 & 0.20 & 0.38 \\
PLKC25-QDot @ 640 nm & 0.03 & 0.44 & 0.20 & 0.33
\end{tabular}

${ }^{a}$ The ratios were calculated using CDPro spectrum analysis program.

important to use the largest and most appropriate reference set. $^{46,47}$ The results from these runs using the largest available reference set are summarized in Table 2.
For the secondary structure analyses, we investigated three different cases in particular: individual peptides, positively and negatively charged peptide pairs interacting each other, and positively charged peptides interacting with negatively charged QDots. First, the secondary structures of each individual peptide were determined. In Table 2, these results show that the percentage of unordered structure overwhelms for positively charged PLKC100 and PLKC25, and the beta strand type secondary structure seems to be dominating for the negatively charged PLE100 and PLE25, possibly also triggering the formation of the $\beta$-sheet structures. Except for PLKC25, the helical content of the peptides is low, which may be a good sign for peptide-peptide interactions because during formation of the peptide film on the silica substrate sheetlike structures are more desirable for exposing the binding sites to immobilize QDots.

In the second case, we examined probable secondary structures of the peptide pairs forming as a result of interactions between them. The peptides were mixed at the same concentrations, following which their circular dichroism data was recorded. We observed for all combinations of the peptide complexes that they become more structured when mixed compared to the individual cases. PLKC100 and PLKC25, each of which possesses alone an almost 50\% unordered type secondary structure, become richer in the beta strand after mixed with PLE peptides. Depending on the increased beta strand content of PLKC after interacting with PLE, it is expected that peptide pairs may transform more into sheetlike structures after their assembly on the silica surface, which is a common case observed for other polypeptide films. $^{48}$

Upon adsorption of the peptide film on a solid surface, a possible rearrangement of the peptide structure is contemplated. In solution, polypeptides mostly exhibit an unordered structure in their native form, which is also the case observed for the peptides we used in this study. This is due to the absence of intermolecular interactions and formation of a stable state. This observation is also common for short peptides screened from phage display libraries for their affinity to bind inorganic surfaces and mineral-forming peptides isolated from organisms. ${ }^{49}$ This is a good indicator that this unordered structure of the peptides in native form possibly increases the binding affinity of the peptides onto the solid surface ${ }^{50,51}$ When the peptides are adsorbed on a solid surface, the core sites for peptide binding are immobilized. This can restrict the free movement of the peptides, and it has been reported that such polypeptide films (e.g., polylysine), as a result, tend to form a sheetlike structure on the solid surface. ${ }^{52}$

Compared to the peptide-peptide interactions, the interaction of peptides with QDots is a completely different situation. During the LbL assembly, the negatively charged CdTe QDots will be positioned against the positively charged PLKC peptides through an electrostatic interaction. In the third case of the secondary structure analyses, to inspect and understand the structural circumstances of these peptides after their interaction with QDots, we extended our observations for the CD characterization to PLKC peptides incubated with CdTe QDots. As a result of their interaction with CdTe QDots, PLKC peptides were found to have enhanced their beta strand type secondary structure elements (reaching a percentage of $44 \%$ in the case of PLKC25 and 38\% in the case of PLKC100). Sheetlike arrangement of these peptides on CdTe QDots surface indicates the possible orientation of the peptides after adsorption.

After the initial characterization of the peptide-peptide and peptide-QDot interactions, the peptide films with/without 

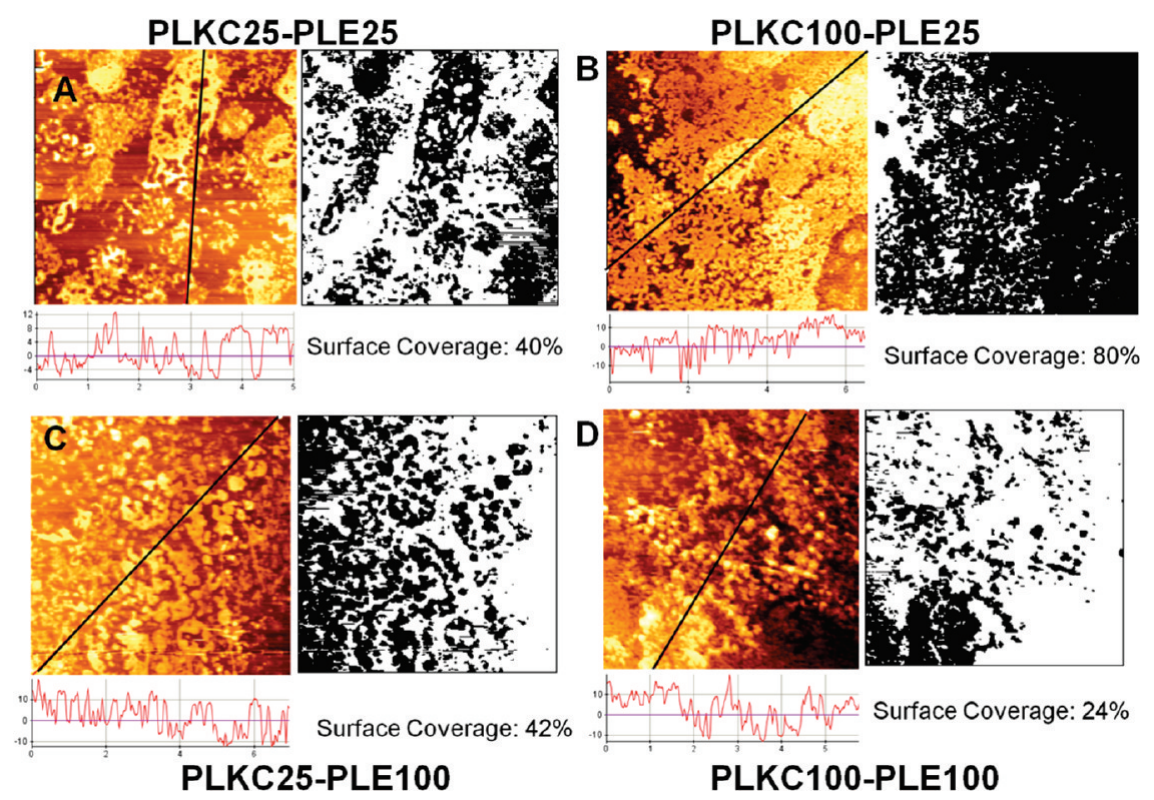

Figure 3. Atomic force microscopy (AFM) images of the peptide-mediated CdTe quantum dot nanocomposite films using different peptide pairs: PLKC25-PLE25 (A), PLKC100-PLE25 (B), PLKC25-PLE100 (C), and PLKC100-PLE100 (D). Black lines indicate the profile lines across the assemblies. The surface fraction images, shown in black and white, represent the surface coverage of the peptide films. The calculated surface coverage of the peptide films is provided below the processed images. The AFM images are taken from a $5 \mu \mathrm{m} \times 5 \mu \mathrm{m}$ area.

CdTe QDots were formed through LbL assembly using a computer-controlled dip coater (Nima Technology, U.K.) operated at a constant dipping and pulling speed. The surface of the silica substrate was preprocessed to increase the negative surface charge density. First a positively charged peptide (PLKC100 or PLKC25) was assembled on the negatively charged silica surface and a negatively charged peptide layer (PLE100 or PLE25) was subsequently laid down, following which another layer of the positively charged peptide was coated. CdTe QDots were then assembled on top of the last positively charged peptide layer. These QDots contain a negative charge on their outer shell due to the thioglycolic acid introduced during their colloidal synthesis (see Supporting Information).

To acquire an idea about the morphology of the resulting peptide-mediated QDot nanocomposites, the films were analyzed using atomic force microscopy (AFM). In Figure 3, the AFM images reflect the assembly and morphology of these QDot-peptide nanocomposite films on the silica surface, for which details of the sample preparation and measurements can be found in the Supporting Information. The surface coverages of these films calculated using an image-processing program called ImageJ are also provided (in percentages) in Figure 3. Although the PLKC100-PLE100 pair exhibited the highest binding energy, the AFM data revealed that this peptide pair did not form a homogeneous and continuous film but instead produced aggregates around QDots that are randomly dispersed. As a result, this nanocomposite film was not spread homogenously on the surface, yielding the lowest surface coverage of $24 \%$ compared to the other films. The PLKC25-PLE25 pair formed a better film, with a surface coverage of $40 \%$, compared to PLKC100-PLE100. However, since their peptide chains are shorter, the formed film consists of islands without a network, which is not a desirable feature for thin-film device applications.

In the case of PLKC25-PLE100, the nanocomposite film also formed islands, which are larger than those of PLKC25-
PLE25 and covered the surface at a ratio of $42 \%$, performing slightly better than the PLKC25-PLE25 pair. Finally, the best film formation was attained by the PLKC100-PLE25 pair, outperforming all the rest by far. This nanocomposite construct with QDots embedded in it was formed in a continuous and homogeneous film on the surface and led to an acceptable surface coverage of $80 \%$, which suffices for most device applications. The resulting PLKC100-PLE25 film contains small spherical particle-like formations, which are interconnected to each other, extending into almost a fully continuous film surface. This molecular architecture of PLKC100-PLE25-assisted CdTe QDot nanocomposite gives us an insight on how to build a robust and homogeneous peptide-mediated film on a solid surface. Subsequently, we analyzed the Förster-type nonradiative energy transfer in these nanocomposite systems, where we focused on the film homogeneity of the nanocomposite constructs as a preferential objective important for obtaining reproducible FRET experiments with controlled FRET efficiency.

Altogether based on the thermodynamic, structural and morphological assessments of these peptide-mediated nanocomposite films, the sheetlike structure of peptides is potentially expected to lead to fibril formation on the solid substrate surface, which has also been suggested in previous studies with amyloid forming peptides. ${ }^{53-56}$ However, due to the chain lengths and strength of the interactions, this does not have to be necessarily always the dominating case. For example, in the case of the longest chain combination, PLKC100-PLE100 formed aggregates instead of fibrils. Such aggregates are considered to prevent the formation of continuous film structures and induce formation of discrete frames of peptide islands. On the other extreme, for the shortest chain combination, PLKC25-PLE25 did not form aggregates, but lacked a continuous film formation. This is attributed to the short chain lengths of the peptide pair, which seem to be insufficient to enable the QDot-peptide complex to form a continuous film. Among the rest of the peptide pairs, in 


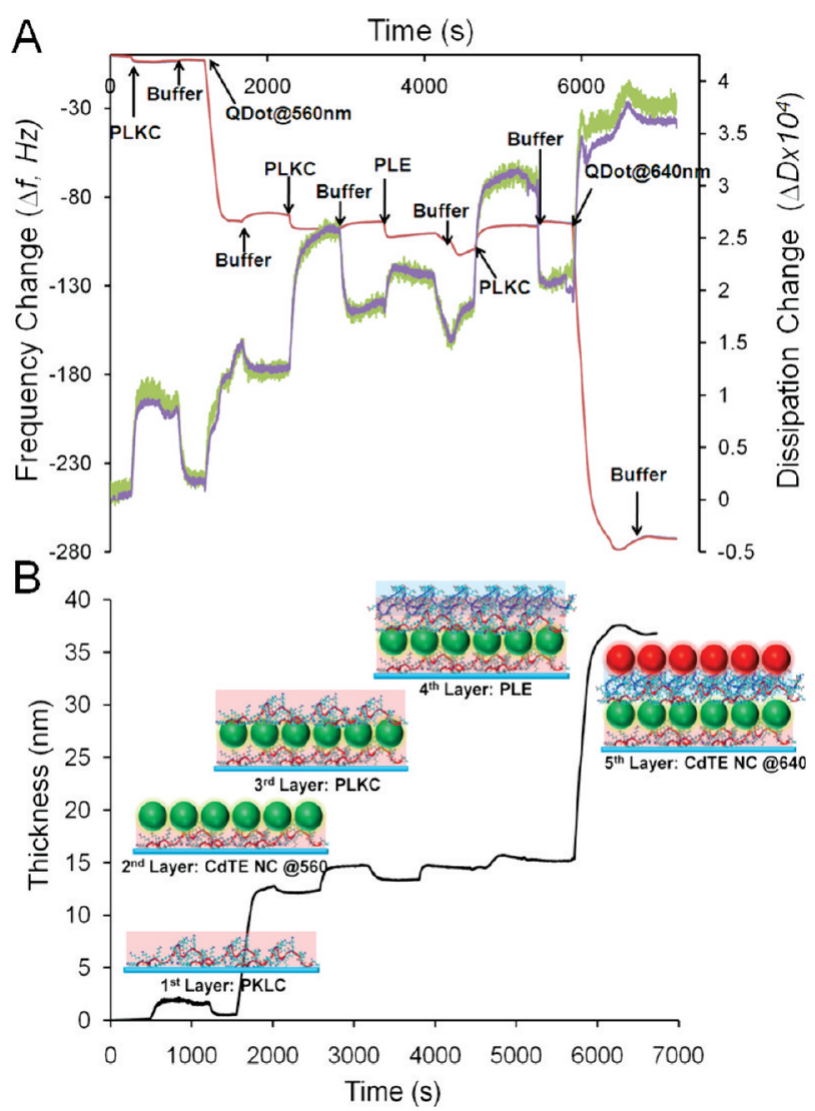

Figure 4. Quartz crystal microbalance with dissipation monitoring (QCM-D), where the red line shows the frequency shift and the green line gives the change in the dissipation upon formation of each layer, and the violet line presents the result of the viscoelastic model fitted to the dissipation data (A). Calculated film thickness from the viscoelastic model for each one of the layer formations during the LbL assembly process (B).

accordance with the ranking of their binding strengths $\left(K_{\text {eq }}\left(\right.\right.$ PLKC100-PLE25) $\gg K_{\text {eq }}($ PLKC25-PLE100) $)$, PLKC100-PLE25 complex allowed for an exceedingly better film formation with respect to the case of PLKC25-PLE100. Here PLKC100-PLE25 is considered to possibly form a sheetlike structure in agreement with their secondary structure, which has been reported to be found in fibril-like biological macromolecular architectures, like amyloidal proteins on solid surfaces. ${ }^{57,58}$ The AFM image shown for PLKC100-PLE25 in Figure 3 also supports the formation of fibril-like extended structures with a homogeneous distribution.

As a special case, we investigated the layer-by-layer film formation in real time using PLKC100-PLE25 peptide pair. With a quartz crystal microbalance with dissipation monitoring (QCM-D) from Q-Sense (Frolunda, Sweden), we tracked the LbL film growth for each layer, and determined the thickness of each film layer using a viscoelastic modeling (see Supporting Information). The assembly plot is depicted in Figure 4, where each step of the assembly is labeled. Here Figure 4A shows the dissipation variation (given in green line) and the frequency shift (given in red line), corresponding to the change in bound mass over time during the LbL assembly process, and Figure $4 \mathrm{~B}$ provides the thickness of each layer calculated using the raw data. These experimental data were fitted using the Voight-based viscoelastic model. ${ }^{59,60}$ In this model the frequency and dissipation changes are related to the formed film thickness, density of the formed film, and viscosity of the film on the quartz resonator. ${ }^{61}$ The film thickness of each layer is obtained by solving the Voigt model at different oscillation frequencies (overtones) of the QCM-D using Q-Tools (version 3.0.7), the software supplied along with the equipment. In Figure 4A, the violet line represents the fitted Voight model estimation to the dissipation data (in green line), with its resulting film thickness extraction for each layer shown in Figure 4B.

The PLKC100 layer adsorbed on the silica surface has a film thickness of $1 \mathrm{~nm}$. Initially, upon adsorption of PLKC100, a large change in the dissipation is observed in Figure 4A. However, this shift decreases dramatically after washing with the buffer. This decrease may result not only from the removal of loosely bound peptides but also from the loss of water held by the peptide in solution. The next layer is CdTe QDots coated on PLKC100 layer by strongly binding them through electrostatic interaction with the surface-bound peptides. Subsequently a series of peptide films are laid down, here PLKC100-PLE25-PLKC100, to make a $3 \mathrm{~nm}$ thick peptide interlayer between the bottom and top QDot layers in the film. This separation is sufficient for nonradiative energy transfer. Finally, on the top is assembled a CdTe QDot layer. Despite the high dissipation alterations observed for the peptide layers, it was found out that the dissipation change is low for CdTe QDot layers during the rinsing phase. This observation is an indicator possibly for the formation of tight QDot films.

Upon study of the architecture of peptide-mediated CdTe QDot nanocomposite films and morphology of the resulting films as discussed above, the molecular constructs of donor and acceptor CdTe QDots were made using polypeptides via $\mathrm{LbL}$ technique. As sketched in the schematics of Figure 4B, first the negatively charged silica layer was decorated with positively charged PLKC100, on top of which the donor QDots emitting at a peak emission wavelength of $560 \mathrm{~nm}$ were assembled. After placing the first CdTe QDot layer serving as the donor, a number of repeating peptide layers (alternating series of PLKC100 and PLE25) were added before the assembly of the last CdTe QDot layer serving as the acceptor on the very top. Such a repetitive use of PLKC and PLE films in an alternating order enables us to create an increasing number of peptide interlayers between the donor-acceptor CdTe QDot films. During the LbL assembly, as shown in Figure 4, each assembly step was followed by an intense washing step to remove nonreacted species from the film surface and achieve a robust and reliable peptidic film.

For these nanocomposite constructs, we first inspected the steady-state photoluminescence (PL) spectra of the peptide films involving the donor alone, the acceptor alone, and the donoracceptor pair. Figure 5 shows the PL spectra taken using a Varian Cary Eclipse fluorescence spectrophotometer, which indicate a clear figure of energy transfer. In Figure 5 three cases are compared: Figure $5 \mathrm{~A}$ shows the photoluminescence from the donor CdTe QDots that emit at $560 \mathrm{~nm}$, while Figure 5B gives the PL from the acceptor CdTe QDots emitting at $640 \mathrm{~nm}$. On the other hand, Figure 5C presents the PL of the donor and acceptor QDot pair together. Figure 5C suggests FRET-based energy transfer from the donor QDots to the acceptor QDots, when compared to Figure 5A,B. In Figure 5C, there is a clear increase observed in the emission intensity of the acceptor QDots (i.e., emission enhancement), whereas there is a clear 
decrease observed in the emission intensity of the donor QDots (i.e., emission suppression). This observation is common for FRET-type interaction between these QDots.

Subsequently we took time-resolved photoluminescence (TRPL) measurements, shown in Figure 6, using a time-correlated photon counting setup (from PicoQuant $\mathrm{GmbH}$, Germany) (see Supporting Information). These were recorded for the QDot-peptide nanocomposite film that contains both the acceptor and donor QDot layers (before and after the protease-based digestion of the peptidic film) and the one that contains only the acceptor QDots in the nanocomposite construct as a reference measurement, all at the acceptor emission wavelength of $640 \mathrm{~nm}$. Also, the protease digestion process of the peptide nanocomposite was presented in the schematics evolving from curves A to D of Figure 6. For the protease cut sketched in Figure $6 \mathrm{~B}$, the nanocomposite film was immersed into a protease solution (from bovine pancreas Type I, Sigma, St Louis, MO, USA) prepared in phosphate buffer ( $\mathrm{pH} 7.4)$ and incubated for 4 $\mathrm{min}$, and the protease was washed off from the surface to take the measurement presented in Figure 6C. The TRPL measurements are compared for three cases: In Figure 6D is the photoluminescence decay measurement of the acceptor CdTe QDots alone in the film as a control group, while in Figure 6A is the lifetime measurement of the CdTe QDot acceptors in the presence of the donor CdTe QDots in the film. The acceptor lifetime was found to be longer (because of a slower photoluminescence decay) in the presence of the donors in the film through FRET-based energy feeding compared to the control group. After the protease

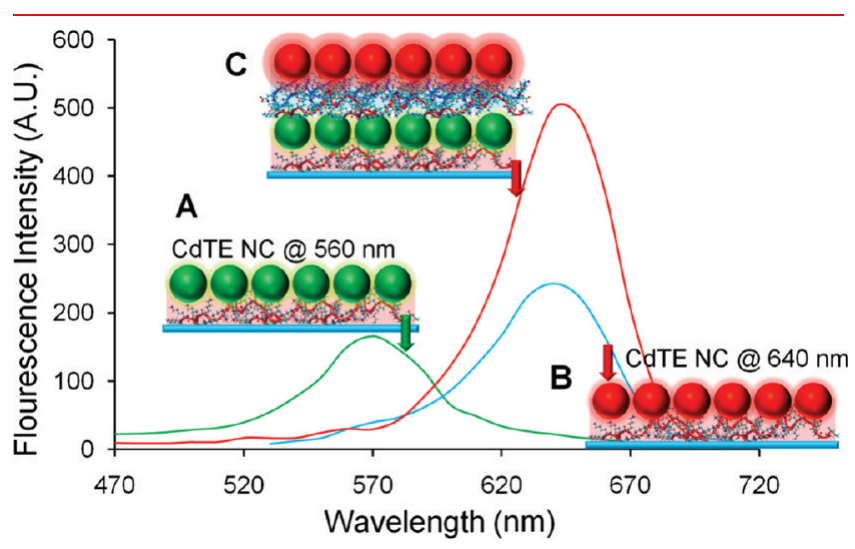

Figure 5. Steady-state photoluminescence (PL) of individual CdTe QDots emitting at $560 \mathrm{~nm}$ (the donor alone) assembled in the peptide film (A), steady-state PL of individual CdTe QDots emitting at $640 \mathrm{~nm}$ (the acceptor alone) assembled in the peptide film (B), and steady-state PL collectively from both CdTe QDots emitting at $560 \mathrm{~nm}$ and those emitting at $640 \mathrm{~nm}$ (the donor-acceptor pair) in the same peptide film, showing the strong modification in the PL intensity of these QDots through FRET (C). treatment, however, the lifetime measurement of the acceptor CdTe QDots in the film given in Figure 6C reveals that the acceptor lifetime is shortened (due to a faster photoluminescence decay with respect to Figure 6A) toward the reference level of the acceptor alone.

The collected TRPL data were fit to a biexponential decay model, which is common for this type of QDot, ${ }^{62,63}$ with a $\chi^{2}$ error close to unity to calculate photon decay lifetimes, using the PicoQuant software supplied along with the instrument, FluoFit. The data fit yields intensity-weighted lifetimes of the acceptor and donor QDots summarized in Table 3. Subtracting the ratio of the donor lifetime in the presence and absence of the acceptors from unity, FRET efficiency $\left(\eta_{\mathrm{FRET}}\right)$ was computed for each of the nanocomposite films. The lifetime analysis suggests that the PLKC100-PLE25 pair keeps the acceptor and donor QDots close enough for the FRET process with a maximum acceptor lifetime of $1.27 \mathrm{~ns}$ and a corresponding $\eta_{\text {FRET }}$ of $\sim 40 \%$, which is a reasonable value for this type of QDot. The PLKC100-PLE25 nanocomposite film, which is considered to provide the best energy transfer case by a notable increase in the acceptor lifetime thanks to the film quality (film homogeneity and reliability), was therefore chosen to further study enzymatic control of the energy transfer with varying peptide interlayer spacings.

The effect of separation between the donor and acceptor $\mathrm{CdTe}$ QDot layers was next tested by varying the number of

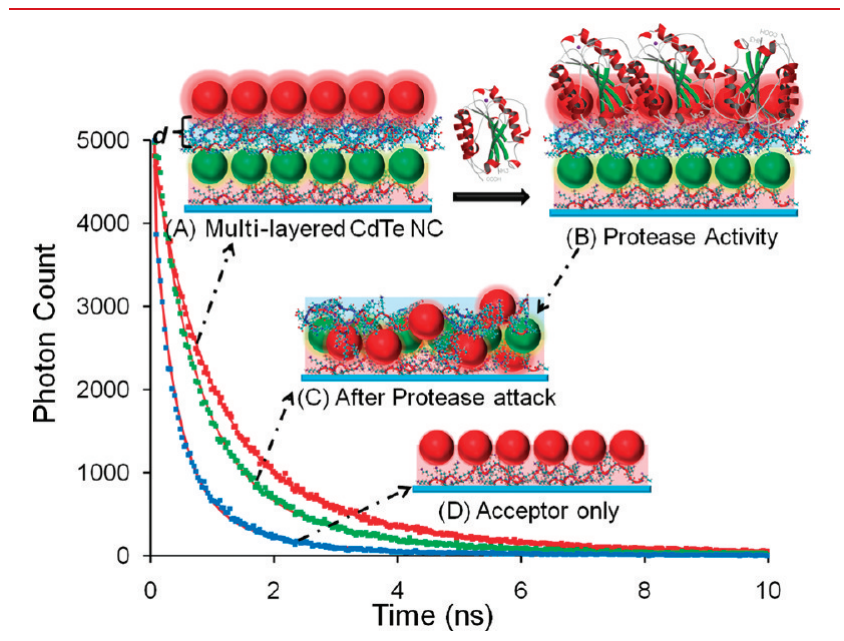

Figure 6. Time-resolved photoluminescence (TRPL) of CdTe QDot donor-acceptor pair with FRET process (A), CdTe QDot donor-acceptor pair after addition of the protease (B), TRPL of CdTe QDot donor-acceptor pair after cleavage of the peptide film (C), and TRPL of CdTe QDot acceptors only (D). The dotted lines are the actual experimental data, while the solid lines are the model fit to the timeresolved photoluminescence data using a biexponential decay model.

Table 3. QDot Donor and Acceptor Lifetimes, Their Decay Rates, and FRET Efficiencies for CdTe QDots Embedded in Different Peptide Films, along with $\chi^{2}$ Error Limits in Their Corresponding Lifetime Analysis

\begin{tabular}{lcccccc}
\multicolumn{1}{c}{ peptides } & $\tau_{\text {acc }}(\mathrm{ns})$ & $\tau_{\text {donor }}(\mathrm{ns})$ & acceptor decay rate $\left(\mathrm{ns}^{-1}\right)$ & donor decay rate $\left(\mathrm{ns}^{-1}\right)$ & $\eta_{\text {FRET }}$ & $\chi^{2}$ \\
single QDots & 0.45 & 0.47 & 2.20 & 3.64 & 8.10 & 1.4 \\
PLKC100-PLE100 & 0.67 & 0.19 & 0.47 & 5.81 & 0.58 \\
PLKC100-PLE25 & 1.27 & 0.27 & 1.79 & 0.40 \\
PLKC25-PLE100 & 0.90 & 0.24 & 1.32 & 5.34 & 0.47 \\
PLKC25-PLE25 & 0.75 & 0.30 & & 1.11 & 1.3 \\
\end{tabular}


repeating peptide interlayers (ranging from three to nine monolayers) between the acceptor and donor layers. Here, up to nine monolayers of interlayer peptides, we were not able to observe a noticeable change in the acceptor lifetimes listed in Table 4. This may be due to the formation of sheetlike structures and strong interaction between the peptides creating a denser and thinner film. If these sheetlike structures were not formed, aggregates would have instead been formed in the film, which could otherwise have caused a significant reduction in the energy transfer rates due to substantially increased separation between the interacting CdTe QDots.

Here since we utilize a peptide-regulated nanocomposite film formation, we can opportunely make use of the advantage to mimic the natural process of biological molecules. In biosystems unwanted proteins and peptides are cleaved by proteases, which effectively serve as a protein scissor, enabling the destruction of poorly folded or contaminated proteins. This approach in nature fits our aim to cut the peptide film between CdTe QDot layers.

Table 4. Change in the Acceptor Lifetime of CdTe QDots Embedded in PLKC100-PLE25 Peptidic Film ${ }^{a}$

$\begin{array}{lccc}\text { no. of monolayers } & \tau_{\text {acc }}(\mathrm{ns}) & \text { acceptor decay rate }\left(\mathrm{ns}^{-1}\right) & \chi^{2} \\ \text { 3 L } & 1.96 & 0.50 & 1.1 \\ \text { 3 L-cut } & 1.16 & 0.85 & 1.2 \\ 7 \text { L } & 1.96 & 0.51 & 1.2 \\ \text { 7 L-cut } & 0.80 & 1.25 & 1.2 \\ \text { 9 L } & 1.84 & 0.54 & 1.2 \\ \text { 9 L-cut } & 1.30 & 0.76 & 1.1\end{array}$

${ }^{a}$ The "cut" refers to the acceptor lifetime after the protease attack.
Figure 7 presents the TRPL experiments for different numbers of the peptide interlayers before and after the protease activity, along with the reference measurement of the acceptors alone. Also, Table 4 shows the lifetimes calculated from these TRPL experiments (for different numbers of the peptide interlayers), all of which exhibit a strong change in the lifetime of the acceptor QDots before and after the protease cut. Upon the enzymatic cleavage of peptides, the peptide film is destructed (see Supporting Information). This causes CdTe quantum dots embedded into the peptide films to be separated from each other. The timeresolved data shown in Figure 7 indicate a faster photoluminescence decay of the acceptor QDots after the protease attack, which corresponds to a reduced energy transfer between the donors and acceptors. This observation supports cleavage of the peptide layers, thereby removal and separation of some of the donor molecules from the film. In the literature a similar approach was used for quenching peptide-linked QDots in solution by other research groups, where the energy transfer was studied using PL measurements, requiring a careful characterization for a quantitative analysis. ${ }^{64}$ Here, the advantage of using the protease cut for the film-based structures is that the ability to control the lifetime of fluorescent nanomaterial constructs based on FRET process by proteolytic enzyme activity allows us to exploit the idea for possible biosensor or photonic device fabrication.

In conclusion, we designed and implemented a biomimetic LbL model to enable and control FRET process between QDots using polypeptides. In this context, two important points were considered with the highest priority, first to create a peptidemediated LbL assembly of QDots in a nanocomposite construct with FRET interaction and second to devise a biomimetic way to

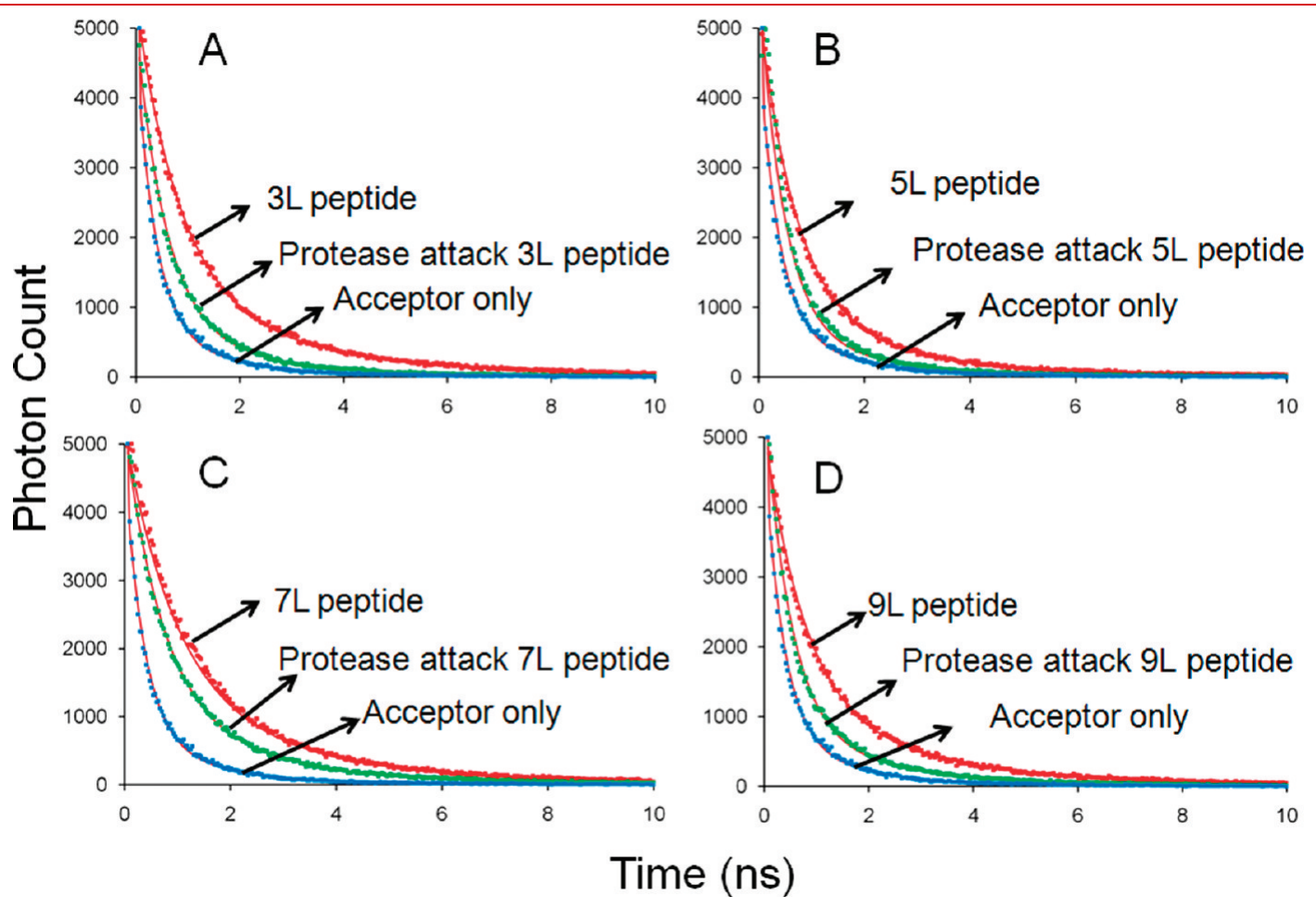

Figure 7. Time-resolved photoluminescence (TRPL) before and after the protease-based cleavage of the nanocomposite films for varying interlayer film thicknesses, with three monolayers of peptide interlayer (A), with five monolayers of peptide interlayer (B), with seven monolayers of peptide interlayer (C), and nine monolayers of peptide interlayer (D). For comparison the cases before and after the protease attack are included along with the reference measurement of the acceptors alone. 
control FRET between QDots. At first place the formulation of a suitable peptide-assisted QDot film using the right pairing of peptides was important. The chain length of peptides, combined with their binding capability and secondary structure elements, controls the morphological properties of the resulting construct. In a direct way these contribute to the determination of film quality. Utilizing this idea, we probed the structural features and binding affinity of the peptide pairs and demonstrated that a strong interaction is not the key for a good film quality alone. Thermodynamic analysis of the positively and negatively charged peptides with different chain lengths yielded $-T \Delta S$ values that are favored during polyelectrolyte peptide interaction. Therefore, a lower entropy system should be chosen with a strong enough binding affinity, which points to the pair of PLKC100PLE25. We supported these findings from the morphology analysis of the peptide films, where we observed the most homogeneous film using PLKC100-PLE25, into which CdTe QDots were also successfully embedded without aggregate formation.

One of the strong motivations for embedding nanocrystals into a peptide-regulated film is to utilize the tools of biochemistry to control FRET effect. With use of protease the peptide layer was cleaved in a controlled manner. So not only the demonstration of FRET control was achieved by such a biochemical process, but also a possible sensor for an enzymatic activity was proposed in the form of a functional peptidic film, which proves to be easy and versatile to use. In currently available, typical fluorescence-based biosensing systems, read-out data rely on the level of optical intensity (thus the collection and counting of emitted photons) and need to be normalized with the greatest care for a reliable quantitative characterization. On the other hand, in our approach, instead of optical intensity levels, the emission kinetics is monitored and photoluminescence decay lifetimes are measured. This leads to a more robust detection, decreasing the possibility of false signal detection, because the changes in the emission kinetics can be correctly measured, despite variations in the optical intensity levels. This approach can be extended by introducing specific protease cleavage sites on polypeptide chains for biosensor applications. Through introducing multiple binding sites for different target molecules, FRET may be controlled and various nanocomposite-based peptidic film biosensors can be devised potentially for a wide range of applications in biological and medical sciences. Also, it could be possible to make sensor arrays using $\mathrm{LbL}$ assembled peptide films to detect different types of proteases (or other targets) in a mixture such as blood samples at the same time. By transfer of this approach to an optoelectronic system, a biocontrolled optical signal transduction can be envisioned. Such peptidebased QDot nanocomposite constructs with FRET interaction promise a wide range of applicability in both biological and physical sciences.

\section{ASSOCIATED CONTENT}

S Supporting Information. The details of preparation of peptide, buffer solutions, and nanocrystals, layer-by-layer assembly, isothermal titration calorimetry, circular dichroism spectroscopy, quartz crystal microbalance, atomic force microscopy, and timeresolved photoluminescence studies, and protease digestion and control experiments. This material is available free of charge via the Internet at http://pubs.acs.org.

\section{AUTHOR INFORMATION}

\section{Corresponding Author}

*E-mail: volkan@bilkent.edu.tr, hvdemir@ntu.edu.sg.

\section{ACKNOWLEDGMENT}

We acknowledge the financial support from Singapore NRFRF-2009-09, ESF European Young Investigator Award (EURYI) Program, and TUBITAK under Grants EEEAG 107E088, 109E002, 109E004, 110E010, and 110E156. H.V.D. acknowledges additional support from the Turkish National Academy of Sciences Distinguished Young Scientist Award, TUBA GEBIP.

\section{REFERENCES}

(1) Carmeli, I.; Lieberman, I.; Kraversky, L.; Fan, Z. Y.; Govorov, A. O.; Markovich, G.; Richter, S. Nano Lett. 2010, 10 (6), 2069-2074.

(2) Hurst, S. J.; Hill, H. D.; Macfarlane, R. J.; Wu, J. S.; Dravid, V. P.; Mirkin, C. A. Small 2009, 5 (19), 2156-2161.

(3) Lee, Y. J.; Yi, H.; Kim, W. J.; Kang, K.; Yun, D. S.; Strano, M. S.; Ceder, G.; Belcher, A. M. Science 2009, 324 (5930), 1051-5.

(4) Li, H. Y.; Carter, J. D.; LaBean, T. H. Mater. Today 2009, 12 (5), 24-32.

(5) Seeman, N. C. Nano Lett. 2010, 10 (6), 1971-1978.

(6) Chen, C. L.; Rosi, N. L. Angew. Chem., Int. Ed. 2010, 49 (11), 1924-1942.

(7) Chen, Y.; Munechika, K.; Ginger, D. S. MRS Bull. 2008, 33 (5), 536-542.

(8) Slocik, J. M.; Tam, F.; Halas, N. J.; Naik, R. R. Nano Lett. 2007, 7 (4), 1054-1058.

(9) Zin, M. T.; Leong, K.; Wong, N. Y.; Ma, H.; Sarikaya, M.; Jen, A. K. Nanotechnology 2009, 20 (1), No. 015305.

(10) Lamm, M. S.; Sharma, N.; Rajagopal, K.; Beyer, F. L.; Schneider, J. P.; Pochan, D. J. Adv. Mater. 2008, 20 (3), 447-451.

(11) Park, H. Y.; Kim, K.; Hong, S.; Kim, H.; Choi, Y.; Ryu, J.; Kwon, D.; Grailhe, R.; Song, R. Langmuir 2010, 26 (10), 7327-7333.

(12) Sapsford, K. E.; Pons, T.; Medintz, I. L.; Higashiya, S.; Brunel, F. M.; Dawson, P. E.; Mattoussi, H. J. Phys. Chem. C 2007, 111 (31), $11528-11538$.

(13) Medintz, I. L.; Uyeda, H. T.; Goldman, E. R.; Mattoussi, H. Nat. Mater. 2005, 4 (6), 435-446.

(14) Ferry, D. K.; Bird, J. P.; Akis, R. Physica E 2004, 25 (2-3), 298-302.

(15) Korbutyak, D. V.; Kalytchuk, S. M.; Geru, I. I. J. Nanoelectron. Optoelectron. 2009, 4 (1), 174-179.

(16) Nizamoglu, S.; Ozel, T.; Sari, E.; Demir, H. V. Nanotechnology 2007, 18 (6), No. 065709.

(17) Demir, H. V.; Nizamoglu, S.; Mutlugun, E.; Ozel, T.; Sapra, S.; Gaponik, N.; Eychmuller, A. Nanotechnology 2008, 19 (33), No. 335203.

(18) Kapanidis, A.; Lee, N. K.; Wang, Y.; Laurence, T. A.; Doose, S.; Michalet, X.; Margeat, E.; Mukhopadhyay, J.; Ebright, R. H.; Weiss, S. Biophys. J. 2005, 88 (1), 660a-661a.

(19) Chen, C.; Peng, J.; Xia, H. S.; Yang, G. F.; Wu, Q. S.; Chen, L. D.; Zeng, L. B.; Zhang, Z. L.; Pang, D. W.; Li, Y. Biomaterials 2009, 30 (15), 2912-2918.

(20) Gonda, K.; Watanabe, T. M.; Ohuchi, N.; Higuchi, H. J. Biol. Chem. 2010, 285 (4), 2750-2757.

(21) Goldman, E. R.; Clapp, A. R.; Anderson, G. P.; Uyeda, H. T.; Mauro, J. M.; Medintz, I. L.; Mattoussi, H. Anal. Chem. 2004, 76 (3), 684-688.

(22) Yang, L. J.; Li, Y. B. Analyst 2006, 131 (3), 394-401.

(23) Kagan, C. R.; Murray, C. B.; Nirmal, M.; Bawendi, M. G. Phys. Rev. Lett. 1996, 76 (9), 1517-1520.

(24) Kagan, C. R.; Murray, C. B.; Bawendi, M. G. Phys. Rev. B 1996, 54 (12), 8633-8643. 
(25) Finlayson, C. E.; Ginger, D. S.; Greenham, N. C. Chem. Phys. Lett. 2001, 338 (2-3), 83-87.

(26) Clapp, A. R.; Medintz, I. L.; Mauro, J. M.; Fisher, B. R.; Bawendi, M. G.; Mattoussi, H. J. Am. Chem. Soc. 2004, 126 (1), 301-310.

(27) Medintz, I. L.; Konnert, J. H.; Clapp, A. R.; Stanish, I.; Twigg, M. E.; Mattoussi, H.; Mauro, J. M.; Deschamps, J. R. Proc. Natl. Acad. Sci. U.S.A. 2004, 101 (26), 9612-9617.

(28) Sapsford, K. E.; Medintz, I. L.; Golden, J. P.; Deschamps, J. R.; Uyeda, H. T.; Mattoussi, H. Langmuir 2004, 20 (18), 7720-7728.

(29) Gokarna, A.; Jin, L. H.; Hwang, J. S.; Cho, Y. H.; Lim, Y. T.; Chung, B. H.; Youn, S. H.; Choi, D. S.; Lim, J. H. Proteomics 2008, 8 (9), 1809-1818.

(30) Ji, X. J.; Zheng, J. Y.; Xu, J. M.; Rastogi, V. K.; Cheng, T. C.; DeFrank, J. J.; Leblanc, R. M. J. Phys. Chem. B 2005, 109 (9), 37933799.

(31) Zhang, C. Y.; Johnson, L. W. Anal. Chem. 2006, 78 (15), $5532-5537$.

(32) Li, M. J.; Xu, X.; Tang, Y.; Guo, Z. N.; Zhang, H. N.; Zhang, H.; Yang, B. J. Colloid Interface Sci. 2010, 346 (2), 330-336.

(33) Kim, G.; Yoon, J.; Kim, J. S.; Kim, H.; Ree, M. J. Phys. Chem. B 2008, 112 (30), 8868-8870.

(34) Decher, G. Science 1997, 277 (5330), 1232-1237.

(35) Franzl, T.; Koktysh, D. S.; Klar, T. A.; Rogach, A. L.; Feldmann, J.; Gaponik, N. Appl. Phys. Lett. 2004, 84 (15), 2904-2906.

(36) Wustman, B. A.; Weaver, J. C.; Morse, D. E.; Evans, J. S. Langmuir 2003, 19 (22), 9373-9381.

(37) Wustman, B. A.; Morse, D. E.; Evans, J. S. Langmuir 2002, 18 (25), 9901-9906.

(38) Poulsen, N.; Sumper, M.; Kroger, N. Proc. Natl. Acad. Sci. U.S.A. 2003, 100 (21), 12075-12080.

(39) Gotliv, B. A.; Kessler, N.; Sumerel, J. L.; Morse, D. E.; Tuross, N.; Addadi, L.; Weiner, S. ChemBioChem 2005, 6 (2), 304-314.

(40) Delak, K.; Collino, S.; Evans, J. S. Biochemistry 2009, 48 (16), 3669-3677.

(41) Seker, U. O. S.; Wilson, B.; Sahin, D.; Tamerler, C.; Sarikaya, M. Biomacromolecules 2009, 10 (2), 250-257.

(42) Robinson, G. W.; Cho, C. H. Biophys. J. 1999, 77 (6), 33113318.

(43) Prabhu, N. V.; Sharp, K. A. Annu. Rev. Phys. Chem. 2005, 56, 521-548.

(44) Makhatadze, G. I.; Privalov, P. L. J. Mol. Biol. 1993, 232 (2), 639-659.

(45) Haynie, D. T.; Zhang, L.; Rudra, J. S.; Zhao, W. H.; Zhong, Y.; Palath, N. Biomacromolecules 2005, 6 (6), 2895-2913.

(46) Sreerama, N.; Woody, R. W. Anal. Biochem. 2000, 287 (2), 252-260.

(47) Sreerama, N.; Woody, R. W. Methods Enzymol. 2004, 383, 318 51.

(48) Haynie, D. T.; Balkundi, S.; Palath, N.; Chakravarthula, K.; Dave, K. Langmuir 2004, 20 (11), 4540-4547.

(49) Collino, S.; Evans, J. S. Biomacromolecules 2007, 8 (5), 1686-94.

(50) Hnilova, M.; Oren, E. E.; Seker, U. O. S.; Wilson, B. R.; Collino, S.; Evans, J. S.; Tamerler, C.; Sarikaya, M. Langmuir 2008, 24 (21), 12440-12445.

(51) Seker, U. O. S.; Wilson, B.; Dincer, S.; Kim, I. W.; Oren, E. E.; Evans, J. S.; Tamerler, C.; Sarikaya, M. Langmuir 2007, 23 (15), 7895-900.

(52) Zhang, L.; Zhao, W.; Rudra, J. S.; Haynie, D. T. ACS Nano 2007, 1 (5), 476-486.

(53) Chimon, S.; Shaibat, M. A.; Jones, C. R.; Calero, D. C.; Aizezi, B.; Ishii, Y. Nat. Struct. Mol. Biol. 2007, 14, 1157-1164.

(54) Liu, G.; Prabhakar, A.; Aucoin, D.; Simon, M.; Sparks, S.; Robbins, K. J.; Sheen, A.; Petty, S. A.; Lazo, N. D. J. Am. Chem. Soc. 2010, 132 (51), 18223-18232.

(55) Lamm, M. S.; Rajagopal, K.; Schneider, J. P.; Pochan, D. J. J. Am. Chem. Soc. 2005, 127 (47), 16692-16700.

(56) Cui, H.; Muraoka, T.; Cheetham, A. G.; Stupp, S. I. Nano Lett. 2009, 9 (3), 945-951.
(57) Knowles, T. P. J.; Oppenheim, T. W.; Buell, A. K.; Chirgadze, D. Y.; Welland, M. E. Nat. Nanotechnol. 2010, 5 (3), 204-207.

(58) Tanaka, H.; Herland, A.; Lindgren, L. J.; Tsutsui, T.; Andersson, M. R.; Inganas, O. Nano Lett. 2008, 8 (9), 2858-2861.

(59) Hook, F.; Kasemo, B.; Nylander, T.; Fant, C.; Sott, K.; Elwing, H. Anal. Chem. 2001, 73 (24), 5796-5804.

(60) Johannsmann, D. Macromol. Chem. Phys. 1999, 200 (3), 501-516.

(61) Vogt, B. D.; Lin, E. K.; Wu, W. L.; White, C. C. J. Phys. Chem. B 2004, 108 (34), 12685-12690.

(62) Chong, E. Z.; Rmatthews, D.; Summers, H. D.; Njoh, K. L.; Errington, R. J.; Smith, P. J. J. Biomed. Biotechnol. 2007, 54169.

(63) Wu, W. Z.; Zheng, Z. R.; Liu, W. L.; Zhang, J. P.; Yan, Y. X.; Jin, Q. H.; Yang, Y. Q.; Su, W. H. Opt. Lett. 2007, 32 (9), 1174-1176.

(64) Shi, L. F.; De Paoli, V.; Rosenzweig, N.; Rosenzweig, Z. J. Am. Chem. Soc. 2006, 128 (32), 10378-10379. 